\title{
Research on Multioperator silk screen printer Based on Communication Protocol Macro
}

\author{
Zhang Zhiming, Sun Jun, Lu Binbin, Duan Yaoshuai \\ School of Electrical \& Mechanical Engineering, Wuhan Textile University, 430073 Wuhan City, China
}

\begin{abstract}
Multioperator silk screen printer is automatic silk screen printing equipment. There are many advantages, such as energy saving, reduce the labor intensity and so on. The control system consists of an intermittent movement main motor and eight color working station printing cycle motion motors. The system controls nine frequency converters through protocol macro communication by using PLC. This paper studies the principle and process characteristics of multioperator silk screen printer. Besides, this paper adopts protocol macro sequence generation method for communication between PLC and frequency converter. This method can control the programming of parameters, such as frequency, operation and monitoring. It also can realize the printing of eight color working stations and monitor the printing status in real time. The practical application proves that the method meets the technical requirements and the reliability is good.
\end{abstract}

\section{Introduction}

The silk screen printing technology is to make silk screen plate with various patterns place on the fabric. Each colour needs a silk screen plate. The active colour paste is added on the silk screen plate when printing. Then the silk screen plate is covered on the fabric. Under the action of the printing scraper and the slurry scraper, the colour paste is applied to the fabric through the gap of silk screen plate. Through the chromatography, a variety of colour patterns become bright colour and rich patterns of finished products [1]. Traditional silk screen printing technology is manual and semi-automatic. The shortcomings are labour intensity, pollution, waste of resources and low printing quality. Therefore, it restricts the development of printing industry in our country more and more [2].

With the development of automation technology, automatic silk screen printing technology began to develop into a cutting edge trend. Automatic silk screen printing technology has the advantages of energy saving, environmental protection, labour intensity reduction and so on. In order to meet the needs of multiple colour printing, independent asynchronous motors are used at each working station. Frequency converters are adopted to adjust speed. The drive uses protocol macros to communicate with multiple frequency converters to control per station printing.

\section{The system principle of multi working stations silk screen printing}

Multi working station screen printing consists of cloth feeding device, centering device, printing device, platen driving device, printing body lifting device and drying and cooling device and so on. The fabric is attached to a plate moving along the radial circulation by a cloth feeding device. Then the fabric is imported printing unit by centering device. When printing, the plate is stationary, the floral board descends and the scraper is scraped. When the printing is completed, the floral board is raised. The fabric moves to next station with the plate and enters the next printing unit.

Multi working station screen printing is controlled by nine asynchronous motors. Eight of them are colour working stations. The working principle is shown in figure 1 .

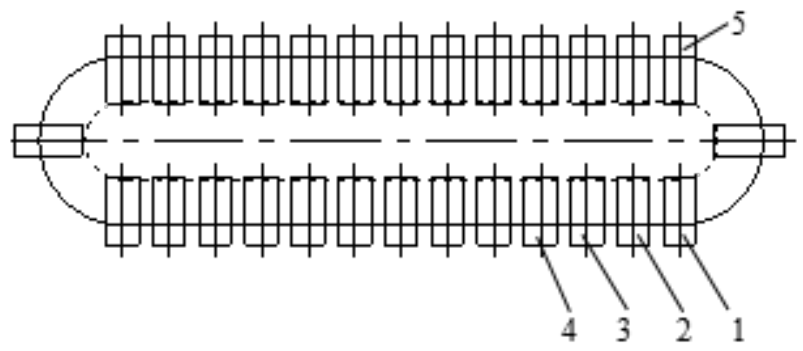

1- Paving position 2- Printing position 3-Drying 4-Cooling 5- Collecting cloth

Figure 1.Working principle of multi working stations silk screen printing

In figure $1,2,3$, and 4 are a combination. They are arranged in eight printing positions in a clockwise order. Each printing position is controlled by an asynchronous 
motor and frequency converter with its speed and steering. Therefore, this system realizes the reciprocating movement of the printing scraper and the slurry scraper to achieve silk screen printing[3]. The actual device is shown in figure 2 .

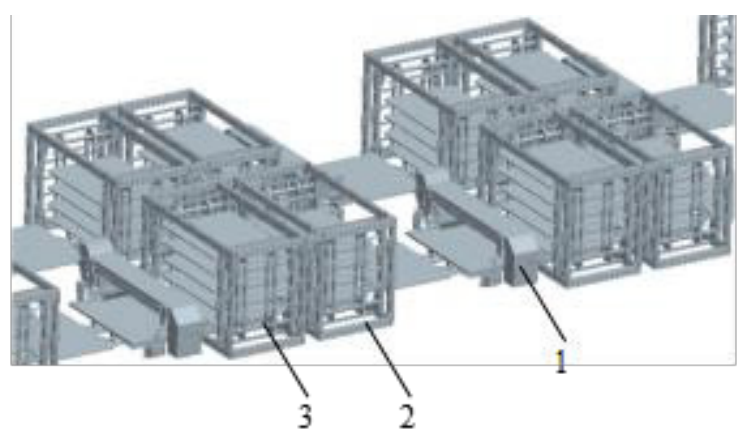

Figure 2. Equipment of multi working stations silk screen printing

\section{The control system of multi working stations silk screen printing}

Multi working stations silk screen printing control system is shown in figure 3. PLC uses $\mathrm{CP} 1 \mathrm{H}$ series $\mathrm{CPU}$ of OMRON. The CPU is equipped with communication module. It has RS-485 communication function. The frequency converter adopts Mitsubishi FR-E500 series, supporting protocol macro programming with PLC. RS485 bus connection is used between slave stations. The use of pneumatic electromagnetic valve can realize the up and down movement of the scraper. So the slurry can be printed on the fabric[4].

Protocol macro is a communication method. It is used to control the PLC and general equipment equipped with RS-232C or RS-422/485 port for data exchange. Through the support of communication protocol macro, a variety of communication equipment for communication protocol can be customized. Therefore, the instructions of the communication protocol macro can be set according to the user's requirements. It also implements an independent definition to send and receive data sequences and communicate with peripheral devices[5].

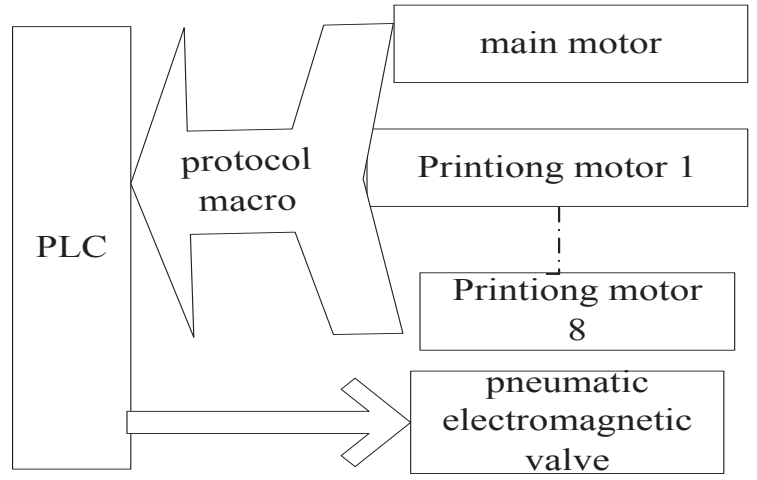

Figure 3. Controlling system diagram of multioperator silk screen printer

\section{The protocol macro system of multi working stations silk screen printing}

The multi working stations silk screen printing protocol macro system mainly consists of three movements: platform intermittent movement, scraper reciprocating printing movement and electromagnetic valve pneumatic movement. Each movement is required to operate in accordance with the specified process. The key point of the control system is how to realize the communication between PLC and frequency converter, send frequency and operation instructions to nine frequency converters and monitor the printing status.

\subsection{Protocol macro communication settings}

In order to make protocol macro communication with PLC, coincident parameters are required to be set up. These parameters include baud rate, stop bit, even-odd check and data length and so on. Only the parameters are consistent, communication can be done. In PLC, they are stored in a special register DM6555 and DM6556 by bit combination. Set the data length to 1 . No even-odd check. The stop bit length is 1 . The baud rate is 9600 .

\subsection{Communication protocol}

Frequency converters and PLC communications use sixteen hexadecimal numbers. Data are automatically transmitted between them using ASCII code. Table 1 is the control code for communication.

Table 1. Controlling code.

\begin{tabular}{|c|c|c|}
\hline Signal & Code & Function \\
\hline ENQ & $05 \mathrm{~h}$ & $\begin{array}{c}\text { Communication } \\
\text { request }\end{array}$ \\
\hline ACK & $06 \mathrm{~h}$ & $\begin{array}{c}\text { Correct } \\
\text { response }\end{array}$ \\
\hline NAK & $15 \mathrm{~h}$ & Error response \\
\hline
\end{tabular}

The definition of data frames stipulates the configuration of data blocks during communication. The communication protocol data request from PLC to frequency converter is shown in figure 4. ENQ is a communication request. The frequency command code is HED. The data account for 4 bits. The sum check code is the 2 ASCII characters represented by the lowest byte of the checked ASCII data sum. The waiting time is the waiting time between the data received from the PLC and the transmission response data.

\begin{tabular}{|c|c|c|c|ccc|c|}
\hline ENQ & $\begin{array}{c}\text { Frequency } \\
\text { converter station } \\
\text { number }\end{array}$ & $\begin{array}{c}\text { Instruction } \\
\text { code }\end{array}$ & $\begin{array}{c}\text { Waiting } \\
\text { time }\end{array}$ & Data & $\begin{array}{c}\text { Sum } \\
\text { check }\end{array}$ \\
\hline 1 & 23 & 45 & 6 & 78910 & 1112 \\
\hline
\end{tabular}

Figure 4. Communication protocol format

\subsection{Create protocol macros}

According to the frequency data frame format of figure 4 , a communication protocol macro can be created in the host computer configuration software. First, set up 
SendMessage and ReceiveMessage information. According to the communication request, check code, data length and data, the structure of sending information and receiving information is generally shown in Table 2 .

Table 2. Data structure of protocol macro

\begin{tabular}{|c|c|c|c|c|c|}
\hline $\begin{array}{c}\text { Identificatio } \\
\mathrm{n} \text { code }\end{array}$ & address & length & data & $\begin{array}{c}\text { Error } \\
\text { checking }\end{array}$ & Terminator \\
\hline
\end{tabular}

The identification code is the data that indicates the beginning of the information. The address is an identifier that sets the node number or indicates that the information sent to the destination. The length is automatically attached to the data length. The data is set for the content of the information. The error check code is setting the SUM, LRC or CRC as the error check code. When sending, the specified error code is automatically appended. When receiving, the error control is automatically carried out according to the error checking code specified in the information. At the same time, the specified amount of data is received. The terminator is the data that sets the end of the information.

For example, in the protocol macro communication sequence, the data for the transmit frequency is $<\mathrm{h}>+$ "00ED07D0" $+<\mathrm{c}>.00$ indicates the frequency converter station number. The ED indicates that the setting frequency is written to the frequency converter RAM. 07D0 stands for that the frequency is hexadecimal. That is, decimal 2000 . The frequency is $20 \mathrm{~Hz}(2000 * 0.01=20)$. $<\mathrm{h}>$ represents the information data header. $<\mathrm{c}>$ represents the data check code.

\subsection{Step settings}

\subsubsection{Repeating counter}

Setting the number of repeated steps, when each step is performed, the counter will add 1 . There are two ways to set up. One way is to reset and the other way is to keep it. If set to reset, once the step is interrupted for some reason, the counter will start counting at the time of next execution. That is, it will still start from 0 . If set to keep it, once the step is interrupted, the counter will still keep the last count and continue to count at the time of next execution.

\subsubsection{Retrial times}

The number of retries can be set to 0-9. It is the number of retries that can be made when an error occurs while sending a command. It is used for the number of retries that can occur after an error has occurred in the transmission.

\subsubsection{Response notices}

If the setting is YES, it means to allow depositing the received data in the third operand of the PMCR instruction or software specified receiving area. The simple point is to allow writing the received data.

\subsubsection{Next step}

There are 4 options: the end, the next step, the direct jump to a certain step and the neglect (That is, END, NEXT, GOTO, ABORT).

\subsubsection{Error handling}

Same as above, there are also 4 options. When an error occurs, it will repeat the work first and retry. If there is still an error, the error will be handled.

\subsubsection{Receive array}

Receive array is another way to receive. It has the function of processing the received data separately. In total, 16 desired reception values can be set. Then when the data is received, the next step can be done according to its requirements. That is, to turn to execute a certain step of the set. When the received data does not meet any of the conditions, the next step will also can be set when the condition is not satisfied.

\subsubsection{Read word (R)}

The "address" or "data" in the sending and receiving information can be set to read word data.

\subsubsection{Wild card $\left({ }^{*}\right)$ and word}

When receiving the data, the amount of data can be set to wild card (*) and word.

\subsubsection{The first-order equation using the variable $\mathrm{N}$}

The first-order equation containing the variable $\mathrm{N}$ is used for importing the address and data. The variable $\mathrm{N}$ will add 1 whenever repeated counters are repeated by a repeated counter specified in the step of a communication sequence.

\subsection{Protocol macro control system implementation}

The protocol macro sets the user communication protocol through the software. Each step can be a send and receive process. In each step, the sending step, the receiving step, the sending and receiving steps can be defined. The data sent and received can be defined completely by the user. Each sequence can perform 16 steps. That is, each sequence can communicate with different devices using different communication formats. In the ladder diagram program, the use of PMCR instructions can easily call different sequences to achieve different communication tasks. Figure 5 is the protocol macro communication program. 


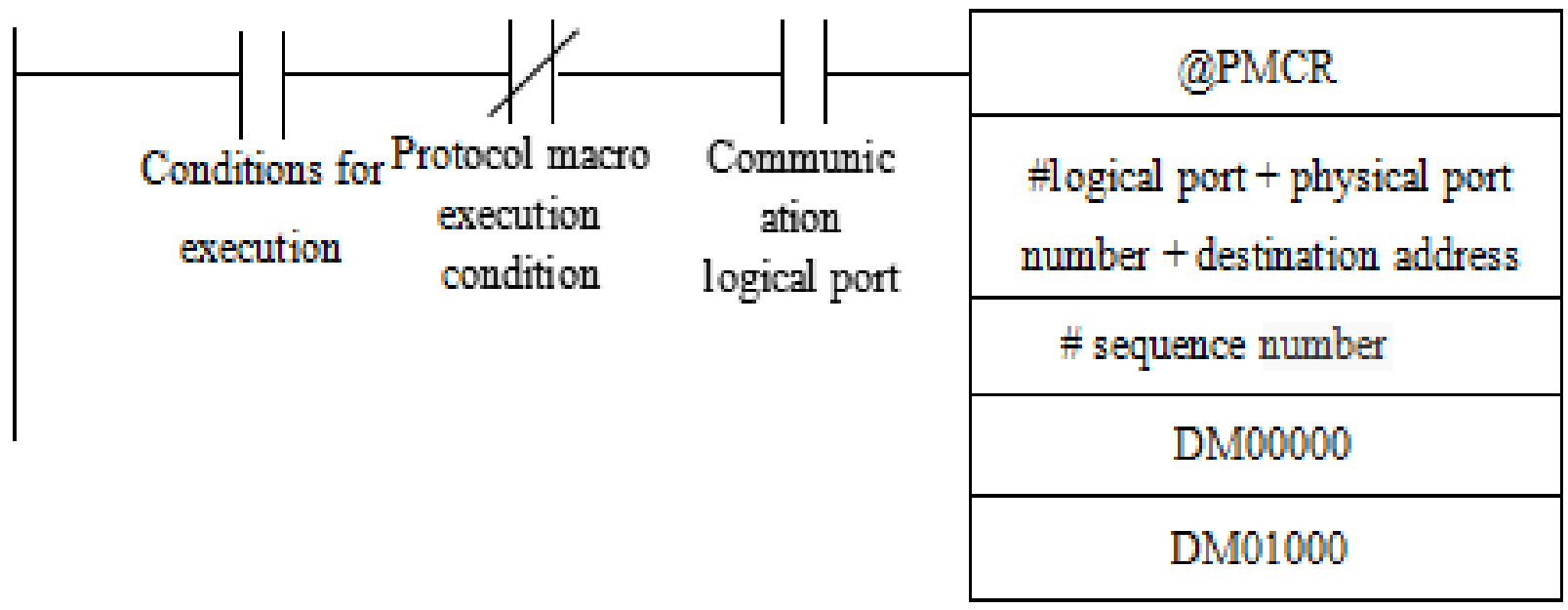

Figure 5. The instruction of protocol micro

The first and second operands of the protocol macro execution instruction are the control word, specified communication port and communication sequence (number 000-999). DM00000 is the first word of the sending data, which is used to store the words of the current send frame. The sending data is stored in the memory area at address DM00000+1. If no data is sent, the number is $\# 0000$. DM01000 is the first word of the received data, which is used to store the words of the received frame. The received data is stored in the memory area at address DM01000 +1 . If no data is received, one memory addresses can be specified at random. The execution of the communication instruction does not overwrite the data of the address.

\section{Conclusions}

According to the requirements of multi working station silk screen printing machine process to the control system, the control system principle based on protocol macro is researched by configuration software of host computer. The communication programs are also developed. Moreover, multicolor printing is achieved. The equipment diagram is shown in figure 6 . The device can simplify the automatic silk screen printing control program, shorten the development time and improve the versatility. The research and practice show that the communication protocol macro realizes multi frequency converter control, simplifies the field wiring and has high versatility and high utilization. The communication protocol macro also creates the conditions for realizing the automation and intelligence of silk screen printing machine, improves work efficiency and reduces labour intensity.

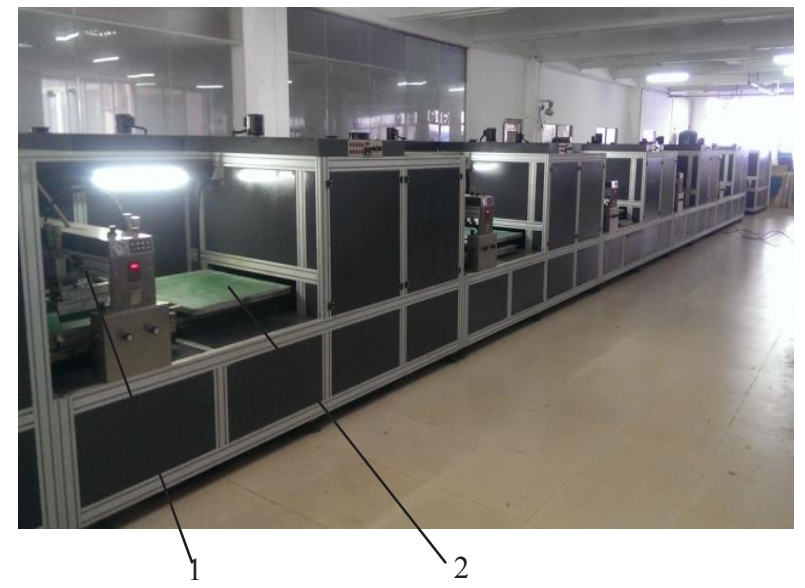

1- Printing mechanism 2-Platform mechanism

Figure 6. The finished equipment

\section{References}

1. Z. M. Zhang, Q. Xu, S. Q. Mei. Journal of Wuhan Textile University, 26, 71,(2013)

2. P. Liu. Journal of Wuhan Textile University,(2011)

3. Y. Q. Liu. Silk screen printing, 8,24,(2013)

4. Z. W. Wang, S. Q. Mei, X. Du. Light Industry Machinery,29,53,(2011)

5. H. Y. Wu, X. N. He, X. D. Lv. Electric Drive, 31,26,(2001) 